\title{
Moving Icons as a Human Interrupt
}

\author{
Colin Ware \\ Joseph Bonner \\ William Knight \\ Rod Cater \\ Faculty of Computer Science \\ University of New Brunswick \\ Fredericton, New Brunswick, Canada
}

This report describes an experiment designed to test the use of simple linear motion as an attention-getting device for computer displays. The experiment involved two tasks: a primary task that required the typed transcription of a document onto a computer screen, and a secondary task that involved detecting and responding to a moving icon signal. The icon consisted of a rectangular bar that grew and shrank in an oscillatory fashion, as the top edge ascended and descended. The amplitude and the velocity of motion were varied systematically and the effect on response time was measured. The results from this secondary task show that there is an inverse relation between the velocity of the moving icon and the response time. No effect was found for amplitude. The speed of the responses suggests that simple motion is an effective attention-getting device for events in the periphery of the visual field.

\section{INTRODUCTION}

In certain situations it is desirable that the user's attention be attracted to a system event. Examples are the arrival of electronic mail, the completion of a background task, or a change in the system state in a real-time process-monitoring application. It is probably safe to assume that the trend towards more sophisticated networking and powerful multitasking workstations will make human interrupt signals a common occurrence at the user interface. Some desirable attributes of a signal

Correspondence and requests for reprints should be sent to Colin Ware, Faculty of Computer Science, University of New Brunswick, P.O. Box 4400, Fredericton, New Brunswick E3B 5A3, Canada. 
event are listed in the following, although it should be recognized that these will vary somewhat from application to application.

1. A signal should be easily perceived even if the user's attention is not directed towards it.

2. If the user should wish to ignore the event while attending to other, more urgent tasks, then the signal or a sequence of signals should continue to remind the user that the event needs attention.

3. The signal should not be so irritating that it makes the computer unpleasant to use.

4. It should be possible to endow the signal with a variety of different levels of urgency.

Methods which have been used to attract attention include sonic beeps or other more ecological sounds (Gaver, 1989), blinking text (Smith \& Goodwin, 1971) or icons that change graphically. In the SunView interface, the arrival of mail is signaled when the flag goes up on the mailbox icon. Unfortunately, this flag is small and the event is easy to miss. In general, static graphical icons in the display will be poor reminders when the user's attention is focused elsewhere on the screen because the vast majority of static visual processing is focused on the fovea, the small area of the visual field where acuity (the ability to discriminate detail) is greatest. For example, $10^{\circ}$ of visual angle from the fovea acuity is less than $30 \%$ percent of acuity at the center of the fovea. However, acuity for moving or blinking visual objects is, under certain conditions, much more uniform across the visual field (Bonnet, 1980). It makes sense that this should be the case because there are obvious evolutionary advantages to the detection of predators and sources of food in the periphery of the visual field. For many animals it would be advantageous to have a background process that constantly monitors the peripheral field for minor movements no matter what is the current focus of attention. This may be the case for humans.

Blinking lights have been shown to be an effective device for attracting attention. In a study relating to shipboard alarm systems, Goldstein and Lamb (1967) showed that subjects were capable of discriminating five flash patterns with approximately $98 \%$ reliability and they responded with an average delay of approximately $2.0 \mathrm{~s}$. A possible penalty to using flashing lights (or blinking cursors) is that much anecdotal evidence suggests that users often find these to be irritating.

An alternative to blinking lights is the use of simple animation in order to attract attention. It seems likely that a smoothly moving icon may be less irritating than a blinking one, although we have only anecdotal evidence to support this. Also, our intuition tells us that there is a natural relation between the velocity or frequency of oscillatory motion and urgency. It may also be possible to encode a number of different types of signals in simple motion paths in order to signal different types of events. Moreover, it has been shown that very simple motions are often interpreted as meaningful in a biological sense (Lethbridge \& 
Ware, 1990; Michotte, 1963), and even given the same interpretation by observers from widely differing cultures (Rimé, Boulanger, Laubin, Richants, \& Stroobants, 1985). This opens the possibility of icon motion, which will be easy to understand and learn. In this study we present the results from a first experiment to investigate whether simple motion can function as an effective human interrupt in a typical task situation.

\section{METHOD}

To investigate the utility of a moving icon as a signal to interrupt user activity we devised a dual-task experiment in which subjects were given the primary task of typing a page of text into a computer terminal. As a secondary task, the subjects were asked to respond by hitting the escape key whenever one or two icons started to move. This caused the icon to stop moving and the subject would resume typing until the next event of this type occurred. The escape key was chosen because of its isolated position on a standard PC keyboard.

\section{Hardware}

An IBM PC with VGA graphics was used.

\section{Stimuli}

The basic screen layout is shown in Figure 1. The screen was divided into a lower window where the text appeared as the subject typed it, and an upper region containing two "icons," one on the upper left and the other on the upper right. The icons consisted of vertical black bars on a white background. The animation of these icons consisted of the oscillatory vertical motion of these bars. The velocity was determined by the number of pixels by which the icon moved on each screen refresh.

We used a temporal antialiasing technique to reduce artifacts which occur when motion is constrained to single pixel units. The basic problem is that, if, for example, the icon is constrained to move by 0.1 pixels per screen refresh, then the result would be a 1 pixel jump every 10 cycles. Thus, what was intended as slow, smooth motion is represented as a series of small jerky movements to which the visual system may well be more sensitive. The antialiasing technique we employed was to compute the proportion of a pixel which should be covered by the leading edge of the icon and give this pixel a grey value proportional to the percentage of the pixel that should be black. In order to do this properly, it is necessary to correct carefully for the fact that most monitors produce a luminance output that is a highly nonlinear function of the input (this calibration, called gamma 


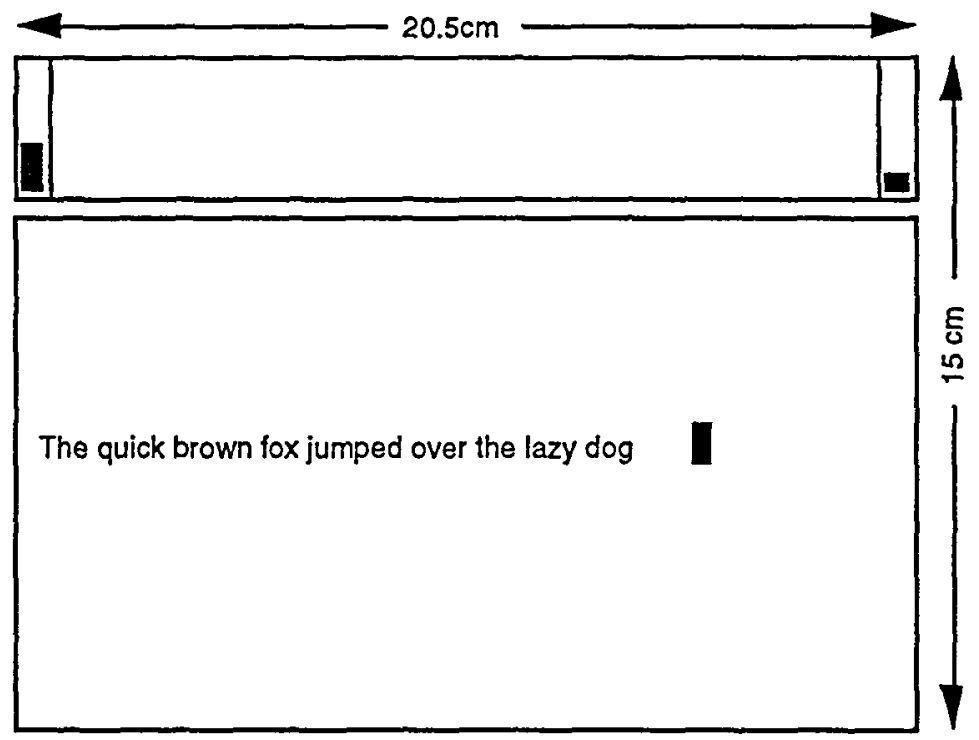

Figure 1. The screen layout is shown approximately to scale. The subject's typing appeared approximately halfway down the lower window. When the typing reached the bottom, the text began to scroll upward to provide more room. The upper right icon is in its resting state. The upper left icon appears as it would part way through its motion cycle.

correction, is described in Cowan, 1983). When gamma correction was done, the motion of our stimulus patterns appeared to be perfectly smooth even in the conditions of slowest motion.

The icon motion was vertical and oscillatory. It consisted of the top edge of the icon growing vertically upward and downward to the extent specified by the amplitude condition. The velocity in a given trial was constant so a plot of position against time would yield a sawtooth function. There were five velocities: 3.0, 6.0, $12.0,24.0$, and $48.0 \mathrm{~mm} / \mathrm{s}$ and three amplitudes: $0.45,0.9$ and $1.8 \mathrm{~cm}$ of motion. The width of the icon was $0.5 \mathrm{~mm}$ and the starting height was $3 \mathrm{~mm}$. The screen luminance was approximately $50 \mathrm{~cm} / \mathrm{m}^{2}$.

\section{Trials}

In a given experimental session there were 5 practice trials followed by 30 test trials. The test trials consisted of one each of all combinations of the five velocities with the three amplitudes with two positions $(5 \times 3 \times 2)$. These 30 test trials were presented in different random order for each subject. The form of each trial was as follows: 
- The subject began entering text, transcribing it from a page placed to the left of the keyboard.

- After an interval randomly determined between 5 and $20 \mathrm{~s}$, the top edge one of the icons began to move.

- The subject, on noticing the motion, pressed the escape key in the upper lefthand corner of the keyboard.

- The icon stopped moving and the subject resumed typing.

A session lasted approximately $15 \mathrm{~min}$. Subjects were asked to regard the typing task as primary. The critical part of the instructions read as follows:

As you type the document, one of the icons will move; when you notice this movement, press the Escape key. Remember, typing the document should be considered to be your primary task, while the moving icon demanding your attention is secondary. Don't worry about your progress through the document-this is not a typing test.

\section{Subjects}

The subjects were 13 adults with varying levels of computer experience and with varying typing skills. However, all of the subjects had some computer experience ranging from several hours to months of text entry.

\section{ANALYSIS}

The response time was log transformed to reduce skewness and lack of homogeneity of variance and an analysis of variance (ANOVA) performed using subject as replication (random effect) and all other effects as fixed. Because a subject performed each particular task (treatment) only once, the error term for the random effects associated with replication and its interactions is nonrecoverable. However, this nonrecoverable term is dominated by the mean square of all interactions of subject and anything else, and subject is statistically significant with respect to any of its interactions at less than .0001 .

\section{RESULTS}

The analysis showed two highly significant main effects: for subject, $F(12,96)=$ $17.47, p<.0001$, and velocity, $F(4,48)=25.83, p<.0001$. None of the other main effects or interactions was significant; thus, no effect was found for amplitude or side of the moving icon. The effect of velocity is summarized by a histogram in Figure 2 and with basic statistics in Table 1. The histogram shows clearly that the 


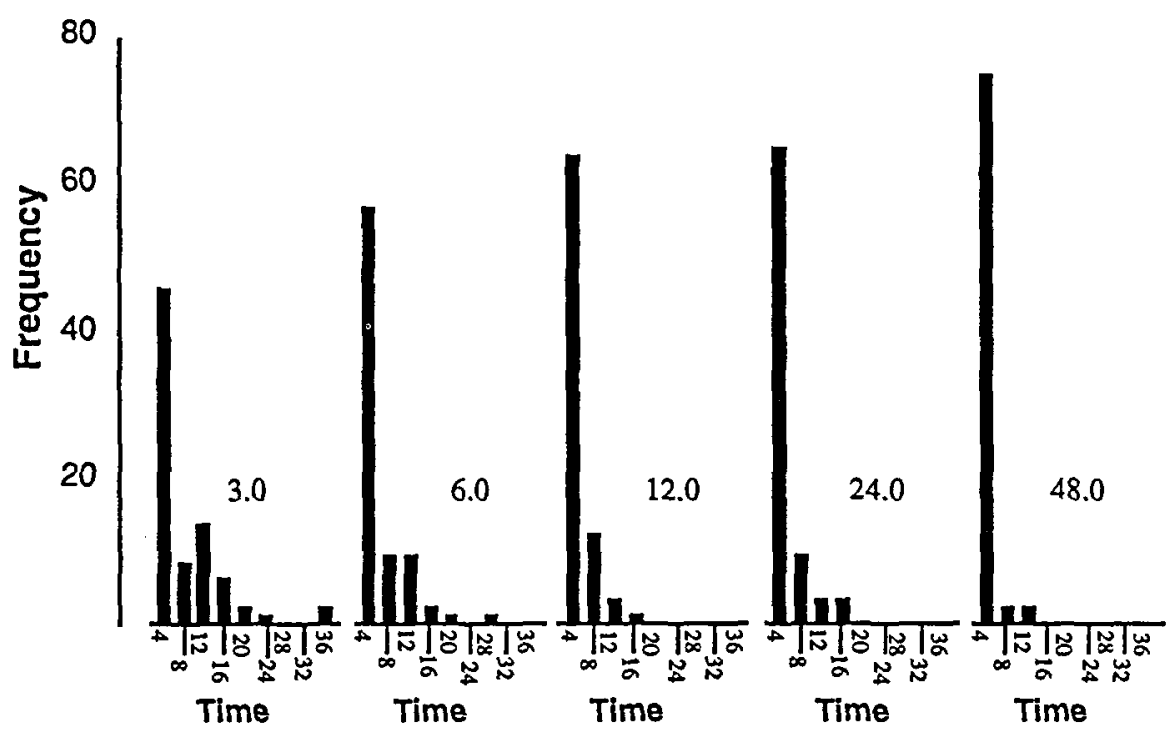

Figure 2. This histogram shows the total number of responses organized in time bins for each of the velocities; the time bins are $4 \mathrm{~s}$ wide.

number of quick responses increases with increasing velocity as the number of long responses diminishes. The mean response time varied from $1.96 \mathrm{~s}$ with the fastest moving icon to $5.31 \mathrm{~s}$ with the slowest moving icon. From a practical standpoint, the fastest and slowest response times are of interest. The fastest response times are all relatively short, showing that subjects would sometimes notice the moving icon rapidly whatever its velocity. The slowest responses are more revealing, they varied from $11.9 \mathrm{~s}$ with the fastest moving icon to $40.3 \mathrm{~s}$ with the slowest. It is interesting to note that these are all roughly five times the mean for each condition. Two other columns in Table 1 show the time thresholds to capture $75 \%$ and $90 \%$ of responses, respectively. Thus, for example, in the $48 \mathrm{~cm} / \mathrm{s}$ velocity condition, $90 \%$ of all responses were made in fewer than $4.4 \mathrm{~s}$. Log response times averaged across subjects appear to be linearly related to the $\log$ of the velocity for the

Table 1. Summary of Responses by Velocities (in $\mathrm{cm} / \mathrm{s}$ ) (All Other Values Are Given in Seconds)

\begin{tabular}{rcccccc}
\hline Velocity & Geometric Mean & Median Point & 75\% Point & 90\% Point & Fastest & Slowest \\
\hline 3 & 5.31 & 5.0 & 11.0 & 16.1 & 1.2 & 40.3 \\
6 & 4.14 & 4.2 & 6.7 & 12.3 & 0.8 & 29.3 \\
12 & 3.08 & 2.9 & 5.0 & 8.9 & 1.1 & 16.5 \\
24 & 3.77 & 2.5 & 4.7 & 8.9 & 0.8 & 17.8 \\
48 & 1.96 & 1.7 & 2.9 & 4.4 & 0.6 & 11.9 \\
\hline
\end{tabular}




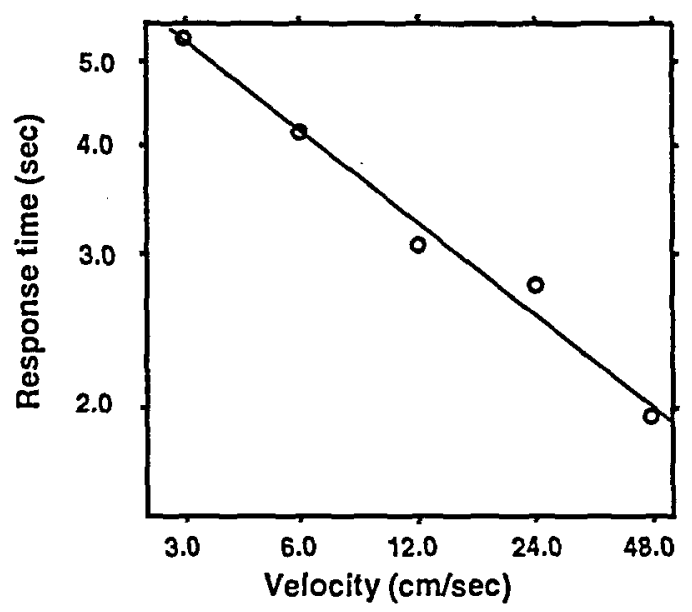

Figure 3. This plot shows the relationship between time to respond and velocity of the moving icon. Note that both axes have logarithmic scales.

range of values tested because the results approximate a straight line on a log-log plot (see Figure 3).

\section{DISCUSSION}

In view of the fact that subjects were instructed to concentrate on typing and only respond to the moving icon as a secondary task, the average $1.96 \mathrm{~s}$ response time in the condition with the fastest velocities seems quite good. It suggests that subjects experienced no trouble in noticing that the icon was moving even though they were not looking directly at it. The longest response times also suggest that simple motion of this kind has practical value as an attention getter; a worst case of $12 \mathrm{~s}$ does not seem excessive, especially in view of the fact that some of the subjects were experienced typists who would habitually finish typing a sentence before attending to the distraction. Thus, our initial supposition that motion makes a good human interrupt has gained some empirical support.

We have only anecdotal information to offer concerning whether a moving icon is irritating; we do not find it so, the smoothness of the motion makes it seem less jarring than a blinking signal.

There is also the issue of the importance of antialiasing. We did it to study a more "pure" form of motion from a psychophysical perspective, but in a practical display it might prove difficult to implement. In this context it is worth pointing out that antialiasing only made a difference for the slowest motion conditions. In the faster conditions there were several pixels of movement with every display 
cycle. Also, the removal of antialiasing would undoubtedly make the pattern more distinct, not less so.

\section{REFERENCES}

Bonnet, C. (1980). Thresholds of motion perception. In A.H. Wertheim, W.A. Wagenaar, \& H.W. Leibowitz (Eds.), Tutorials on motion perception. New York: Plenum.

Cowan, W.B. (1983). An inexpensive scheme for calibration of a color monitor in terms of CIE standard coordinates. Computer Graphics, 17, 315-321.

Gaver, W.W. (1989). Audotory icons: Using sound in computer interfaces. Human-Computer Interaction, 2, 167-177.

Goldstein, D.A., \& Lamb, J.C. (1967). Visual coding using flashing lights. Human Factors, 9, 405-408.

Lethbridge, T., \& Ware, C. (1989). A simple heuristically based method for expressive stimulus-response animation. Computers and Graphics, 13, 297-303.

Michotte, A. (1963). The perception of causality. New York: Methuen. Rimé, B., Boulanger, B., Laubin, P., Richants, M., \& Stroobants, K. (1985). The perception of interpersonal emotions originated by patterns of movements. Motivation and Emotion, 9, 241-260.

Smith, S.L., \& Goodwin, N.C. (1971). Blink coding for information display. Human Factors, 13, 283-290. 